Commun: Communication et organisation

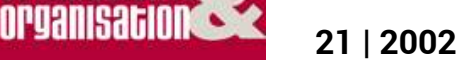

Espace et communication dans les organisations

\title{
Espace, communication et ingeniérie concourante
}

Arlette Bouzon

\section{OpenEdition}

Journals

Édition électronique

URL : http://journals.openedition.org/communicationorganisation/2623

DOI : 10.4000/communicationorganisation.2623

ISSN : 1775-3546

Éditeur

Presses universitaires de Bordeaux

Édition imprimée

Date de publication : 1 mai 2002

ISSN : 1168-5549

Référence électronique

Arlette Bouzon, «Espace, communication et ingeniérie concourante », Communication et organisation [En ligne], 21 | 2002, mis en ligne le 19 décembre 2012, consulté le 19 avril 2019. URL : http:// journals.openedition.org/communicationorganisation/2623; DOI : 10.4000/ communicationorganisation.2623

Ce document a été généré automatiquement le 19 avril 2019

(c) Presses universitaires de Bordeaux 


\title{
Espace, communication et ingeniérie concourante
}

\author{
Arlette Bouzon
}

1 L'espace de travail renvoie à un questionnement qui va bien au-delà des préoccupations classiques de gestion des activités organisationnelles tentant de rentabiliser le coût des mètres carrés utilisés. L'espace est aussi un lieu de la vie au travail qui agit sur les comportements individuel et collectif des employés. Il fixe le cadre d'interaction dans lequel s'inscrivent les actions humaines (Goffman, 1992) et est susceptible d'être modifié, adapté, voire détourné par l'individu ou le groupe. Mais le terme même d'espace est ambivalent, et aussi présent que mal défini. Contrairement à celui de territoire qui constitue une aire visible et stable, le concept d'espace se définit comme une frontière invisible autour de la personne (Fischer, 1990). Plus qu'un lieu, qui correspond à un endroit concret, matériel, défini (Lautier, 1999), l'espace définit les conditions de vie des individus. Avec la prise en compte de la proxémic, il peut être compris comme un langage (Hall, 1971) qui révèle l'organisation et. le cas échéant, ses dysfonctionnements.

2 Cet article tente d'appréhender le rôle dévolu à l'espace et son influence dans une activité de conception de type ingénierie concourante. Il se fonde sur certains résultats d'une enquête réalisée dans des entreprises du secteur spatial, ces trois dernières années, portant sur la place de la communication dans le processus de conception innovante. Une centaine d'entretiens compréhensifs ont été menés au cours de cette étude auprès des différents acteurs de la conception de satellites et sondes interplanétaires, complétés par une observation sur le terrain quand elle était possible.

3 Après avoir rappelé les différentes façons de considérer l'espace, nous présenterons brièvement l'ingénierie concourante, et analyserons la manière dont l'espace et son aménagement peuvent donner lieu à une dynamique sociale dans une activité collaborative. Nous illustrerons notre propos par l'un des cas particuliers observés. 


\section{L'espace dans l'organisation}

4 La question de l'espace dans l'organisation recouvre les conditions d'attribution de l'espace personnel dans un espace collectif. Ce partage de l'espace influence le fonctionnement de l'organisation en favorisant ou en limitant les interactions entre ses membres et en différenciant ces derniers. Aussi son aménagement est-il l'objet de multiples négociations dans l'entreprise et d'une réflexion plus ou moins approfondie par les gestionnaires qui considèrent cet espace comme une ressource parmi d'autres à optimiser. Mais selon quel critère? Quelle est, en effet, la fonction que l'organisation assigne concrètement à l'espace: lieu de vie? Facteur de communication entre les acteurs ? Facteur de performance? Ou simple contrainte génératrice de coût?

\section{Espace et communication}

5 Il n'est certes pas possible d'isoler l'espace de la culture dans laquelle il intervient (Grawitz, 1986, p. 116). Les distances interpersonnelles varient fortement, par exemple, entre les sociétés occidentales et asiatiques (Hall 1971, 1984). Mais au-delà des spécificités nationales et des contextes particuliers, l'espace de travail peut être considéré comme une production de l'entreprise, elle-même ouverte sur un environnement qui l'influence (Morgan, 1986), et qui évolue au cours du temps. Ainsi, s'opposant à une longue tradition de bureaux fermés, le bureau paysager naquit en Allemagne dans les années soixante et ne fut introduit que tardivement en France, après un détour par les États-Unis. Mais ce type d'aménagement nouveau, censé favoriser l'ouverture tout en minimisant les surfaces utilisées, fut largement rejeté dans notre pays en raison de particularismes culturels (Fischer, 1997). Depuis le début des années quatre-vingt, l'aménagement de l'espace s'inspire fréquemment de deux modèles architecturaux voisins: l'atrium et la rue intérieure. L'atrium, qui se rencontre fréquemment dans les pays anglo-saxons, se caractérise par l'existence d'un lieu central important d'où sont issus des axes de circulations distribués vers des espaces sociaux proches censés favoriser la vie sociale. Plutôt adoptée dans les pays d'Europe du Nord, la rue intérieure, appelé aussi «combioffice », est fondée sur l'idée d'une communauté villageoise qui met en avant la demande évoluée de communication dans l'organisation et témoigne de l'apparition de valeurs nouvelles dans un monde organisationnel instable (Duffy, 1992; Treiber, 1989). Plus récemment, des bureaux non-assignés (accessibles en libre-service) ont fait leur apparition en raison des coûts d'exploitation élevés de certains locaux de prestige. Mais cette répartition spatio-temporelle de l'espace attribué aux acteurs, initiée notamment par l'entreprise Andersen Consulting, reste encore très confidentielle.

6 Le choix même des locaux apparaît déterminant et le fait d'adopter une architecture horizontale ou verticale conditionne à l'avance les formes de communication à venir. Certaines études ont montré que les schémas de circulation ont un effet direct sur la communication, en favorisant notamment les rencontres de hasard (Allen, 1977). Selon Grannovetter (1982), la capacité d'un individu à obtenir des informations issues d'entités éloignées dans l'organisation dépend du nombre de ses liens faibles (par opposition à des liens de nature fonctionnelle ou hiérarchique). Stone et Luchetti (1985) ont par exemple travaillé sur le modèle des petits bureaux individuels rassemblés autour d'un espace ouvert. Cet agencement donne à l'utilisateur l'isolement nécessaire pour travailler de 
manière concentrée, sachant que, quand il veut communiquer, il peut sortir dans l'espace ouvert conçu à cet effet. Les attentes, plus ou moins explicites des différents acteurs en matière d'intégration et de différenciation ont également fait l'objet de diverses recherches. Pour certains auteurs, l'espace a la fonction de résoudre les tendances centrifuges de l'organisation en favorisant la création d'une structure équilibrée. Lautier (1999) considère par exemple les nouvelles organisations architecturales comme un moyen d'atténuer les différents champs de tensions de l'organisation, issus des forces contradictoires qui la composent.

7 Dans ce contexte, les entreprises cherchent à exploiter l'utilisation des mètres carrés de manière optimale, en tentant à la fois de limiter les coûts y afférents et de favoriser des conditions de travail performantes. Mais si l'espace influence l'utilisateur et son comportement, les relations entre ce cadre de vie et le fonctionnement organisationnel sont toutefois encore mal connues. Le lien entre espace et efficacité par exemple n'est pas direct et ne peut guère être mesuré compte tenu des influences multiples s'exerçant sur ces deux dimensions.

\section{De l'espace ressource à l'espace résidu}

8 Si aucune théorie ne renseigne précisément, à notre connaissance, sur les liens entre espace individuel et collectif, pratiques communicationnelles et fonctionnement organisationnel, il existe néanmoins dans les organisations des pratiques sociales qui se développent et changent au fil du temps; la difficulté de penser l'espace de travail n'empêchant pas les entreprises d'en produire. Cette gestion du cadre professionnel envisage l'espace comme une « ressource » que l'entreprise doit exploiter au mieux pour supporter ses activités. Ainsi des réalisations audacieuses, telles que le siège de l'entreprise néerlandaise Centraal Beheer ou le centre Challenger de l'entreprise Bouygues, ont vu le jour sous l'impulsion de dirigeants résolus. Mais l'espace se révèle bien souvent, dans les faits, comme le résultat d'un compromis entre diverses contingences matérielles et les exigences des différents groupes d'acteurs en présence. Il est généralement déterminé après que d'autres décisions jugées plus importantes aient été prises par les décideurs. Ainsi, les contraintes liées à l'utilisation de l'espace sont-elles multiples. Indépendamment des budgets disponibles, la configuration des locaux ne peut pas suivre l'évolution de l'organisation au jour le jour. Les bâtiments existants ont une capacité d'adaptation limitée et de nouvelles constructions imposent des délais incompressibles. Le respect de consignes de sécurité et des nonnes en vigueur limite les possibilités d'agencement. L'implantation des prises électriques, des câbles téléphoniques et informatiques et de la climatisation, transitant le plus souvent via des planchers ou fauxplafonds, conditionne l'installation des ordinateurs eux-mêmes régis par certaines considérations ergonomiques. En outre, de même que les mobiliers qui l'occupent (qualité et taille des bureaux, épaisseur des moquettes...), l'espace lui-même constitue un signe visible de différentiation dans un univers hiérarchisé (dimension, nombre de fenêtres, proximité des lieux de décisions...) dont l'importance symbolique prime souvent sur toute autre considération. Les alternatives envisageables s'en trouvent limitées, et l'espace devient alors un « résidu ».

9 Ecartant la question de l'efficacité organisationnelle liée à l'espace (le caractère composite d'une organisation ne permettant pas de montrer précisément l'apport de l'espace sur sa production), nous nous intéressons en revanche à la manière dont 
l'aménagement de l'espace peut donner lieu à une dynamique sociale, notamment dans le cadre d'une activité de conception de type ingénierie concourante.

\section{L'ingénierie concourante}

10 D'origine anglo-saxonne et mise au point pour le développement de produits nouveaux dans des industries de masse, l'ingénierie concourante (dont la traduction française fait référence au verbe concourir), appelée aussi simultanée, fait l'objet d'un certain engouement parmi les dirigeants d'entreprises. Partant du constat que l'exécution séquentielle des phases de définition du produit, de définition du processus de fabrication puis de réalisation du prototype était longue et supportait difficilement les rétroactions, cette approche relativement nouvelle consiste à rechercher un fort recouvrement entre les différentes phases de conception pour permettre d'éventuels retours immédiats sur celles-ci et raccourcir la durée globale du processus de développement (Darses, 1997). Elle suppose l'intégration des divers spécialistes concernés dans le processus de conception (marketing, étude, méthode, fabrication, exploitation), et implique une étroite coopération des différents acteurs qui sont invités à travailler ensemble dans des espaces de réflexion commune.

11 Suivant les secteurs d'activité où elle s'est développée, l'ingénierie concourante a donné naissance à différentes méthodes de travail en groupe. Ainsi, chez les constructeurs automobiles, la notion de "plateau » correspond à un lieu, espace de travail, où sont rassemblées les équipes du constructeur et celles de ses différents fournisseurs pour concevoir puis produire ensemble un produit de grande série. L'introduction de cette nouvelle manière de travailler s'est accompagnée d'une profonde modification des relations et activités de chacun en clarifiant les interfaces; les fournisseurs de pièces détachées devenant progressivement responsables à part entière de sous-systèmes complets (avec prise de risques, investissements mais aussi valeur ajoutée plus conséquents). Dans le groupe projet de la Twingo par exemple, qui constitua l'une des premières expériences de ce type menées en France, les séparations physiques antérieures, du fait de la "féodalité métier », ont été remplacées par un espace de travail unique qui en rapprochant les acteurs, a augmenté les contacts informels, diminué les circuits d'information, et rendu l'équipe-projet plus performante notamment en raison de la "matérialisation visuelle de ce qui était en jeu ( (Midler, 1993). Dans le domaine aéronautique, la «maquette virtuelle » est réalisée par de puissants outils de simulation (CAO) pour donner à tous les acteurs d'un projet, quel que soit leur métier, une vision complète de l'état de la conception du produit au fur et à mesure de son développement. Enfin dans le secteur spatial, le "Team X", notion issue d'outre-Atlantique, rassemble dans un même lieu, et généralement pendant de courtes périodes, des spécialistes de tous les métiers concernés (mathématicien, automaticien, informaticien, électricien, mécanicien, thermicien,...) pour travailler ensemble sur un projet et tenter de faire émerger les solutions les plus globalement satisfaisantes : l'ingénierie coopérative allant bien au-delà de la simple gestion des interfaces.

Outre le temps gagné en ne mettant plus les divers processus en série, l'ingénierie concourante permet de lutter contre le cloisonnement dans les organisations en favorisant les échanges réactifs. "Sa vitalité repose sur la création de nombreuses passerelles transversales de communication, sur l'intensification des échanges d'information entre les spécialistes dès les premières phases du projet. Une telle 
organisation a le mérite d'accélérer les temps de développement et de réduire notablement les gaspillages" (Gramaccia, 2001, p. 174). La modification des pratiques engendre des interactions nouvelles qui rendent le travail plus efficace. En effet, l'optimisation d'un produit découle rarement d'une optimisation séparée de ses constituants mais résulte surtout du meilleur compromis trouvé entre les caractéristiques de ces derniers par l'ensemble des spécialistes. «Dans un tel mode de travail, tous les métiers interviennent non pas séquentiellement mais ensemble tout au long du projet. Se voient alors les conséquences des décisions des uns et des autres et les individus abordent les questions au fur et à mesure qu'elles se posent. » (Grosjean, Lacoste, 1999, p. 196). Mais si l'ingénierie concourante peut accélérer les processus de développement tout en améliorant le produit lui-même (en termes de satisfaction de la demande de l'utilisateur au meilleur coût), elle implique un certain recouvrement des savoirs et des langages pour permettre la communication effective quel que soit l'espace d'échanges retenu. Elle nécessite également une forte implication des acteurs et notamment des experts (pas toujours disponibles) et n'est pas exempte de méthodologie et de coordination, ce qui donne au chef de projet un rôle d'animateur déterminant. Elle suppose notamment la validation immédiate de certains résultats, afin qu'une erreur de l'un ne mette pas en péril le travail des autres ; la coordination des actions étant beaucoup plus lâche et moins formalisée en raison de l'instantanéité.

L'importance de l'espace dans ce nouveau type de conception apparaît prégnante. L'analyse de Midler (1993) concernant la conception de la Twingo est tout à fait éloquente à ce propos. La transformation de l'espace de travail initialement fondée sur la séparation géographique par un plateau rapprochant physiquement les individus a facilité les compromis entre les différents acteurs et a permis in fine l'obtention de résultats significatifs en termes de délai et de qualité des solutions choisies. Cette forte interaction souhaitée ne peut se développer que s'il existe de nombreuses possibilités d'échanges directs entre les acteurs; la communication relationnelle s'avérant plus déterminante que la communication fonctionnelle dans la recherche d'idées nouvelles (Girin, 1987). Aussi, l'espace n'est-il plus seulement considéré comme une ressource passive dont les entreprises cherchent à baisser les coûts, mais devient-il une dimension communicationnelle essentielle permettant les échanges entre acteurs et participant au brassage des populations; ce dernier favorisant l'innovation, l'émergence d'une logique commune de projet, l'entraide mutuelle et les effets d'apprentissage.

Mais si les objectifs assignés à cet espace sont relativement clairs, sa nature est multiple et son utilisation sur le terrain diffère souvent de celle qui était attendue. Nous illustrerons notre propos par un exemple d'ingénierie concourante menée dans le secteur spatial que nous avons étudié.

\section{L'espace dans un cas d'ingénierie concourante}

15 Nous avons été amenés à observer une équipe de conception chargée de travailler sur un projet de microsatellites; une conception de type ingénierie concourante ayant été retenue en raison de l'originalité du projet associée à de fortes contraintes de délai. 


\section{L'observation sur le terrain}

16 Fortement influencée par l'orientation nouvelle «bester, faster, cheaper» donnée par Richard Godwin, l'administrateur de la NASA, et après un séminaire sur l'ingénierie concourante organisé dans l'entreprise par des consultants ayant travaillé sur des projets similaires Outre-Atlantique (Team X), la direction décida, en 1998, la constitution d'une équipe d'une vingtaine de personnes devant concevoir ensemble une plate-forme de petits satellites, de manière simultanée. Compte tenu des enjeux techniques et économiques, les responsables du projet se sont d'abord et surtout préoccupés du recrutement des acteurs (animateur, ingénieur système, concepteurs, experts métiers), des choix méthodologiques (analyse fonctionnelle, analyse de la valeur, analyse préliminaire de risques...) et de la mise en place des moyens adéquats (ordinateurs, logiciels...). Les choix portant sur l'espace sont intervenus ensuite. Cherchant à regrouper dans un même lieu l'équipe de projet et les représentants du client, afin d'accélérer les échanges et faciliter l'émergence d'idées nouvelles, la direction réussit à libérer, dans un bâtiment existant, une grande salle rectangulaire $\left(60 \mathrm{~m}^{2}\right)$, qu'elle équipa, à sa périphérie, d'une vingtaine de postes informatiques reliés en réseau avec sièges pivotants, ainsi que d'une grande table de réunion installée en son centre, munie de tableaux, de micros et de rétro et vidéo projecteurs. Cet espace collectif ne répondait toutefois pas exactement à la demande initialement formulée par le chef de projet qui souhaitait un local «en fer à cheval », de cent à cent-cinquante mètres carrés, comprenant deux salles et un « coin détente ».

17 L'équipe de conception prit possession de ces locaux, mais après une période d'adaptation relativement peu structurée, les responsables durent se rendre à l'évidence, ces locaux restaient vides la plupart du temps, ne servant qu'à quelques réunions. Les acteurs censés y travailler en permanence ne s'y rendaient que de manière épisodique, restant dans leurs bureaux antérieurs respectifs le reste du temps.

18 La direction tenta de remédier à cette situation en corrigeant certains dysfonctionnements mineurs liés à l'aménagement des locaux et en cherchant à convaincre les intéressés des avantages que pouvaient procurer les investissements réalisés. Mais après plusieurs tentatives infructueuses de réorganisation du travail collectif, un compromis fut trouvé: chacun continuerait de travailler comme il l'entendait, sachant que cet espace dévolu à l'ingénierie concourante devait être utilisé le plus possible... tout au moins pour les réunions d'articulation.

\section{Tentative d'explication}

19 La situation des acteurs diffère selon les moments dans le processus de conception, et avec lui les conditions d'utilisation de l'espace : «Certains sont engagés dans des activités de co-conception, tandis que d'autres participent à des activités de conception distribuée. Ces deux situations peuvent être rencontrées au cours d'un même processus de conception et peuvent également être successivement prises en charge par un même acteur » (Darses, Falzon, 1996, p. 126). Dans la co-conception, les étapes du raisonnement sont réparties entre les partenaires qui développent conjointement la solution. Ils partagent un but identique à atteindre et chacun contribue à sa résolution collective avec ses compétences spécifiques. Celle-ci concerne soit un même métier, impliquant par 
exemple un spécialiste du projet et un expert consulté pour résoudre un problème particulier, soit la frontière entre divers métiers notamment pour en définir les interfaces. Le mécanicien, le thermicien et l'automaticien s'associeront, par exemple, pour résoudre un problème lié à des phénomènes thermoélastiques sur un générateur solaire. Dans la conception distribuée, en revanche, les acteurs qui « sont simultanément (mais non conjointement engagés) sur le même processus de coopération accomplissent des tâches bien déterminées, celles-ci ayant été allouées préalablement, et poursuivent donc des buts (ou du moins des sous-buts) qui leur sont propres tout en ayant pour objectif de participer le plus efficacement possible à la résolution collective du problème. » (Darses, Falzon, 1996, p. 127). La coordination des activités distribuées entre les acteurs s'effectue par le chef de projet généralement secondé par un médiateur technique, «l'ingénieur système, qui a notamment pour rôle de gérer les interfaces en termes de contraintes et performances attendues.

Ces parts respectives d'activité de co-conception et de conception distribuée diffèrent sensiblement selon le type d'organisation du travail choisi. Cependant, la majeure partie de l'activité de conception en ingénierie concourante d'un système complexe, telle que celle que nous avons observée, reste encore distribuée entre des acteurs spécialisés même si elle implique une activité de co-conception plus conséquente (environ trente pour cent du temps) que dans une conception de type séquentiel. Des périodes de fortes interactions s'avèrent nécessaires pour permettre une synchronisation cognitive entre les individus, notamment durant les premières phases du projet (lors de la définition des interfaces...) et quand des problèmes surviennent (au cours des essais...).

21 La co-conception est beaucoup plus facile quand les individus sont regroupés dans un espace commun où ils peuvent échanger, réfléchir ensemble, se coordonner et efficacement coopérer... Au sein d'un tel "cadre de participation» (Goffman, 1987), chacun peut laisser flotter son attention au gré de ses préoccupations, sans regard mutuel, ni action commune. Mais si un problème surgit ou une question est posée, sa réflexion peut se focaliser rapidement et s'organiser autour d'un centre d'intérêt commun selon son rôle et sa spécialité : « dès qu'un mot est prononcé, tous ceux qui se trouvent à portée de l'événement possèdent par rapport à lui un certain statut de participation » (Gofffman, 1987, p. 9).

En revanche, le besoin de communication est tout différent quand il s'agit d'une activité distribuée, à mener seul ou au sein d'un groupe restreint. Les acteurs éprouvent alors le besoin de s'isoler, à la fois pour eux-mêmes et pour leur travail, surtout quand leurs capacités de concentration influencent directement leur activité. Si l'espace ouvert permet cette "grand-messe perpétuelle " souhaitée par les promoteurs de l'ingénierie concourante, les individus souffrent aussi du regard des autres et leur travail connait parfois un rendement médiocre en raison des nombreuses distractions ambiantes et des nuisances visuelles ou acoustiques. L'exposition immédiate d'idées encore brouillonnes est souvent mal vécue par des concepteurs préférant l'autocensure au risque d'énoncer des propositions erronées ou simplement mal présentées. Des banalités indiscutables sont alors plus facilement échangées que de véritables originalités qui ne seront exprimées qu'après certaines vérifications auprès d'un public restreint et sûr, apte à la confrontation technique sans engendrer de jugement sur la personne. La disponibilité à tout moment devient également un facteur de stress et le surpeuplement peut conduire au « renfermement psychologique». Le repli sur soi devient alors une condition pour pouvoir travailler, ce qui va à l'encontre de la nature même de l'ingénierie concourante. 
ertains acteurs interrogés estiment avoir du mal à « lâcher leur bureau » initial, dont la possibilité d'ouvrir ou de fermer la porte permet l'isolement. Ainsi l'appropriation des nouveaux locaux ne s'est-elle apparemment pas réalisée en raison de leur incapacité à offrir un espace propice à la concentration, mais sans doute aussi à cause du besoin éprouvé par chacun de disposer d'un espace qui lui soit propre; le bureau indiquant en partie la place de l'individu dans l'entreprise. L'espace rassemble en effet les individus en les situant les uns par rapport aux autres, sur les plans fonctionnel, hiérarchique ou selon toute autre signification plus ou moins explicite, telle que l'appartenance à différents réseaux. Or le bureau non assigné du nouvel espace ne donnait pas la possibilité de s'approprier un bout d'entreprise, de constituer un territoire bien à soi, une «basearrière » pour affronter plus aisément la vie organisationnelle. Cet enracinement joue un rôle dans le prolongement de l'identité de l'individu par les objets (Becker, Steele, 1995), considérés par certains chercheurs comme l'expression de leur position dans l'organisation (Stone, Luchetti, 1985).

\section{Conclusion}

Plus l'organisation du travail se révèle complexe, plus l'influence de l'espace ne peut être comprise qu'en regard des activités précises qui s'y déroulent, sans négliger ce qu'il implique d'imaginaire symbolique et de négociation sociale. Ainsi, dans le cas d'ingénierie concourante que nous avons observé, l'activité était très diversifiée et les périodes de réelle conception collective ne représentaient qu'une part limitée du temps de travail. Des besoins antagonistes, de communication, d'isolement, et d'appropriation d'un territoire individuel, ont été à l'origine de tensions que la solution retenue n'a su qu'imparfaitement résoudre.

Une politique volontariste considérant l'espace comme une ressource à optimiser pour agir sur la dynamique sociale s'avère délicate et ne donne pas obligatoirement de meilleurs résultats que ceux obtenus à partir d'un espace résidu, fruit des multiples décisions organisationnelles; les acteurs concernés s'étant appropriés ce dernier et l'ayant intégré dans leurs logiques de fonctionnement. De plus, le développement des technologies de l'information et de la communication ont imperceptiblement modifié les distances spatiotemporelles entre les acteurs qui peuvent travailler ensemble sur des objets communs sans sortir de leurs bureaux respectifs. En effet, le contact physique direct ne semble plus avoir l'importance qu'il avait au moment des premières réflexions sur l'ingénierie concourante, même s'il facilite la convivialité et la coopération.

Cette réflexion s'inscrit dans un questionnement plus large, portant sur la question du déterminisme de l'espace. D'un coté, l'espace est perçu comme contraignant l'individu à adopter certains comportements, de l'autre, il est considéré comme une forme d'expression de la société parmi d'autres et qui suit son développement. Cette distinction nous parait toutefois dépassée et nous amène à considérer plutôt l'espace comme un cadre d'interaction qui fixe des limites aux acteurs en même temps qu'il leur laisse des possibilités d'action à l'intérieur de ces limites. L'espace est alors envisagé comme le résultat d'une négociation sociale, un enjeu organisationnel et humain et un atout pour la communication.

Communication et organisation, 21 | 2002 


\section{BIBLIOGRAPHIE}

ALLEN T.-J., Managing the Flow of Technology. Cambridge/Mass. : MIT Press, 1977.

BECKER F., STEELE F., Workplace by Design. Mapping the High-Performance Workscape. San Francisco : Sage, 1995

CHANLAT J-F.,L'individu dans l'organisation : les dimensions oubliées. Paris : Editions Eska, 1990.

DARSES F., L'ingénierie concourante : un modèle en meilleure adéquation avec les processus cognitifs de conception. In BOSSARD P., CHANCHEVRIER C, LECLAIR P., Ingénierie concourante : De la technique au social. Paris : Economica, 1997, p. 39-55. DARSES F., FALZON P., La conception collective : Une approche de l'ergonomie cognitive. In TERSSAC G. de, FRIEDBERG E., Coopération et conception. Toulouse : Octares, 1996, p. 123-137.

DUFFY F., The Changing Workplace. Londres : Phaidon Press limited, 1992. EVETTE T., LAUTIER F., De l'atelier au territoire : le travail en quête d'espaces. Paris : L'Harmattan, 1994.

FISCHER G.-N., Psychologie des espaces de travail. Paris : Armand Colin, 1989.

FISCHER G.-N., Psychologie de l'environnement social. Paris : Dunod, 1997.

GIRIN J., Le siège vertical. Vivre et communiquer dans une tour de bureaux. Gérer et comprendre, décembre 1987, n 9, p. 4-14.

GOFFMAN E, Façons de parler. Paris : Minuit, 1987.

GRAMACCIA G., Les actes de langage dans les organisations. Paris : L'harmattan, 2001.

GRANNOVETTER M., The strength of Weak Tics. A Network Theory revisited. In MARSDEN P., LIN N., Social Structure and Network Analysis. NY : Sage, 1982.

GRAWITZ M., Méthodes des sciences sociales. Paris : Dalloz, 1986.

GROSJEAN M., LACOSTE M., Communication et intelligence collective. Le travail à l'hôpital. Paris : PUF, 1999.

HALL E. T., La dimension cachée. Paris : Seuil, 1971.

HALL E. T., Le langage silencieux. Paris : Seuil, 1984.

HOTIER H., Non verbal et organisation. Paris : L'Harmattan, 2000.

LACOSTE M., Interaction située et dimension collective du travail. In SIX F., VAXEVANOGLOU X., Les aspects collectifs du travail. Toulouse : Octares, 1990.

LAUTIER F., Ergotopiques : sur les espaces des lieux de travail. Toulouse : Octares, 1999.

LETESSIER J., Dessine-moi un bureau virtuel. Architecture Créée, déc.95/jan, 1996, n 269.

MORGAN G., Images de l'organisation. Paris : Eska, 1986.

MIDLER C, L'auto qui n'existait pas. Management de projet et

transformation de l'entreprise. Paris : InterEditions, 1993.

NACHER Y., Architecture \& Images D'entreprise : nouvelles identités. Liège : Mardaga Editeurs, 1990. 
STONE P., LUCETTI R., Your office is where you are. Harvard Business Review, mars-avril 1985, p. 102-117.

TREIBER D., Norman Foster et la tour de la Hong Kong Bank. Paris : METL, 1989.

\section{RÉSUMÉS}

Cet article s'intéresse à l'espace utilisé par les acteurs au cours d'une activité de conception de type ingénierie concourante. Fondé sur les résultats d'une enquête réalisée dans des entreprises du secteur spatial, il montre, notamment à partir d'un cas observé, qu'une politique volontariste considérant l'espace comme une ressource à optimiser pour agir sur la dynamique sociale ne donne pas toujours les résultats escomptés. L'espace se révèle en effet le résultat d'une négociation sociale, qui cherche à répondre à des besoins parfois antagonistes, de communication, d'isolement, et d'appropriation d'un territoire par les individus.

This article deals with the space used by the designers during an activity of concurrent engineering. Based on the results of an investigation carried oui in companies of the space sector. it shows that a policy considering the space as a resource to be optimised in order to act on social dynamics does not always give the anticipated results. The space indeed appears the result of a social negotiation. which seeks to meet sometimes antagonistic needs. of communication, isolation, and appropriation of a territory by the individuals.

\section{INDEX}

Mots-clés : espace, ingénierie concourante, conception, co-conception, conception distribuée, interaction

\section{AUTEUR}

\section{ARLETTE BOUZON}

Arlette Bouzon, Maître de conférences, habilitée à diriger des recherches, à l'Université de Toulouse III, au département Information-Communication. Chercheur au LERASS (Laboratoire d'Études et de Recherches appliquées en Sciences sociales), participe aux travaux de l'équipe de recherche MICS (Médiations en Information et Communication Spécialisées) et du groupe Org\&co portant sur la communication organisationnelle de la SFSIC. Thèmes de recherche : communication autour du risque, conception dans les organisations. 